\title{
LEIBNIZ Y EL MÉTODO DE LA METAFÍSICA: EL DEBATE CON DE VOLDER ACERCA DE LA DEFINICIÓN DE SUSTANCIA
}

Rodolfo E. Fazio

UBA-CONICET

\begin{abstract}
RESUMEN: En nuestro trabajo estudiamos el debate entre Leibniz y De Volder acerca de la naturaleza de la sustancia. En particular, argumentamos que a pesar de no encontrarse en la correspondencia un argumento a priori a favor de la definición de sustancia como fuerza primitiva activa, Leibniz presenta una justificación de la misma en otros términos. En primer lugar, analizamos la prueba a priori a favor de las fuerzas vivas y criticamos su validez para la metafísica. En segundo lugar, examinamos la defensa que Leibniz lleva adelante de su definición y evaluamos el procedimiento que subyace a ella.
\end{abstract}

PALABRAS CLAVES: Leibniz; sustancia; método; fuerza primitiva; metafísica.

ABSTRACT: In this paper we study the debate between Leibniz and De Volder concerning the nature of substance. We claim that, despite the absence of an a priori proof for his definition of substance as primitive active force, Leibniz defends his notion in other terms. First, we analyze the a priori argument for the living forces and we criticize its validity in metaphysics. Secondly, we examine Leibniz's notion of substance and evaluate the method that underlies his position.

KEYWORDS: Leibniz; substance; method; living force; metaphysics.

"A muy pocos se les concede que, aun diciendo cosas verdaderas, les sean aceptadas de inmediato; además, lo verdadero no siempre es lo verosímil".

Leibniz a De Volder ${ }^{1}$

\section{Introducción}

Desde sus primeros escritos Leibniz muestra un gran interés por determinar qué procedimiento ha de seguirse en metafísica para establecer la

1 GP II, p. 181. 
verdad de sus proposiciones. Siguiendo el espíritu de otros pensadores de la época, en sus años juveniles (1663-1672) confía en la posibilidad de que el método geométrico sea capaz de encauzar a la filosofía primera, al igual que hiciera con la filosofía natural. Esta posición, empero, es criticada y abandonada por el filósofo alemán durante su estancia en Paris (1672-1676). En efecto, en el marco general de la crisis que constituye el período parisino y en paralelo a sus reflexiones críticas respecto de las tesis fundamentales de su metafísica, Leibniz se ocupa de un tema más básico: su método. En particular, en estos años sospecha de la utilidad del modelo geométrico que algunos modernos han propuestos para tratar cuestiones de filosofía primera. En el breve opúsculo De vera methodo philosophiae et theologiae ac de natura corporis (1673-1675), Leibniz explicita la deplorable situación en la que se encuentra la metafísica si se la compara con disciplinas tales como la matemática o la física. En efecto, estas últimas ciencias han sido encauzadas y comienzan a mostrar sus frutos, por ejemplo, en la construcción de máquinas que hacen más agradable y fácil la vida de los hombres. En este breve opúsculo Leibniz confiesa que en su juventud creyó, junto con la mayoría de los filósofos modernos, que el more geometrico era también el camino a seguir a fin de poder dirimir las cuestiones metafísicas:

\footnotetext{
Habiendo apartado mi espíritu de los muy rigurosos estudios sobre los cánones sagrados y el derecho divino y humano hacia las disciplinas matemáticas, degusté la dulzura de una doctrina seductora y me adherí rápidamente a esos escollos de sirena". ${ }^{2}$
}

${ }^{2}$ AA VI 3, p. 155. Durante su juventud Leibniz queda cautivado por la idea de aplicar el método de los geómetras en cuestiones metafísicas. En particular, esto se percibe luego de su paso por la Universidad de Jena en 1664. Allí conocerá a Erhard Weigel, de quien recibe la idea de conciliar la metodología 


\section{Dossiê Leibniz, Dissertatio - Volume Suplementar 03 UFPel [2016]}

Leibniz admite que en sus primeros años adopta la metodología de los modernos con la esperanza de que ésta brinde los mismos beneficios en filosofía primera que los que diera en las otras disciplinas. Sin embargo, las investigaciones parisinas son las responsables de liberarlo de tal encantamiento.

Por un lado, Leibniz es consciente de los graves problemas que tiene la metodología adoptada por la escolástica. En particular, enfatiza el hecho de que los argumentos ofrecidos por los filósofos de la Escuela no conducen sino a interminables disputas entre las partes. Por otro lado, los modernos adjudican esta falencia al procedimiento utilizado, pues la falta de rigor en la argumentación sería lo que imposibilita que la filosofía llegue a buen puerto. Como solución estos últimos proponen, entonces, la aplicación del método geométrico en la metafísica. En De vera methodo philosopbiae et theologiae Leibniz critica esta conclusión. En principio, reconoce con los nuevos filósofos que, sea cual sea el método de la escolástica, éste ha fracasado. Sin embargo, juzga que el problema de la filosofía clásica no es la falta de rigurosidad argumentativa, sino algo más básico: el punto de partida de sus pruebas, es decir, el establecimiento de las definiciones. Al respecto señala:

Existen quienes piensan que el rigor matemático no tiene lugar por fuera de las ciencias que vulgarmente se llaman matemáticas. Pero ellos ignoran que es lo mismo escribir matemáticamente que razonar formalmente, como dicen los lógicos [...]. De hecho, los escolásticos trabajaron con un único

aristotélica con el procedimiento matemático. En el apéndice de la Dissertatio de arte combinatoria (1666) encontramos, por ejemplo, una primera prueba de la existencia de Dios - de corte cosmológico - que procede a partir de definiciones, postulados y axiomas. Sobre este tema, cf. Moll (1978), p.74. 
vicio, pues, aunque razonaron la mayor parte del tiempo con suficiente orden o, como digo, matemáticamente, dejaron el uso de las palabras a lo incierto. De allí que en lugar de una única definición había muchas definiciones, en lugar de una demostración sólida había muchas argucias nacidas de ambas partes, por las cuales sus dogmas divinos y sus contemplaciones a menudo dignas de admiración fueron purgadas sin dificultad por los hombres educados matemáticamente. ${ }^{3}$

Leibniz cree que los razonamientos escolásticos son rigurosos, pero fallan al no haber establecido adecuadamente las definiciones de las que parten. Los filósofos modernos se equivocan en su diagnóstico y, por ello, también en su remedio. En efecto, el método geométrico parece solucionar el asunto en la medida en que fija definiciones precisas y a partir de ellas emprende derivaciones. Sin embargo, Leibniz cree que con ello no se soluciona el problema de la escolástica, sino que sólo se la oculta detrás de una aparente mayor rigurosidad argumentativa, puesto que el problema que aqueja a la metafísica no es determinar qué se sigue de la noción de sustancia o de la de cuerpo, sino en verdad establecer qué es sustancia, qué es cuerpo, etc. Y esta falencia afecta tanto a los antiguos como a los modernos ${ }^{4}$. De este modo, aun cuando estos últimos han observado correctamente las consecuencias negativas de los métodos escolásticos, la aplicación del método matemático en cuestiones

\footnotetext{
${ }^{3}$ AA VI 3, p. 156.

4 Leibniz juzga que hay ciertos conceptos que han sido tomados sin los recaudos suficientes tanto por la filosofía como por la teología. Este tema guarda una relación directa con sus estudios sobre el infinito propios del período parisino, los cuales le enseñaron que es posible pensar cosas que, no obstante, una vez analizadas se muestren como contradictorias. Este inconveniente afecta tanto al método escolástico como al de los modernos: permitir la introducción de conceptos sin realizar un análisis correcto de ellos que pruebe su consistencia.
} 


\section{Dossiê Leibniz, Dissertatio - Volume Suplementar 03 I UFPel [2016]}

propias de la metafísica sólo disfraza el problema bajo una mayor apariencia de rigurosidad. El objetivo primero en metafísica ha de ser, entonces, establecer las definiciones. En nuestro trabajo trataremos una definición particular en un caso concreto: la caracterización de la sustancia en el debate Leibniz-De Volder (1698-1706). Además de atender al contenido de la discusión entre ambos filósofos, nos interesaremos por comprender el modo específico en que Leibniz presenta su definición de sustancia en términos de fuerza primitiva activa.

\section{El caso de la definición de sustancia en el debate con De}

\section{Volder}

Durante los años que siguen a la crisis parisina, Leibniz retoma la idea de que la actividad es la marca distintiva de la sustancia. Sin embargo, a partir de 1677 en adelante presenta una novedosa comprensión de la misma, signada en parte por sus estudios en el campo de la física. En sus primeras publicaciones sobre metafísica, a saber, De primae philosophiae emendatione et de notione substantiae (1694), el Système nouveau de la nature et de la communication des substances (1695) y el Specimen dynamicum (1695), define a la sustancia como fuerza activa primitiva. Cabe señalar que, hasta este momento, Leibniz sólo había utilizado el concepto de fuerza en sus escritos de dinámica, en los que defiende que la fuerza motriz de los cuerpos (esto es, el efecto que son capaces de producir) es distinta de la cantidad de movimiento y, de un modo más problemático, que tal potencia está ínsita en ellos. Sin embargo, el concepto propio de la dinámica no es en principio 
equiparable al que se utiliza en metafísica, sino que sólo constituye el punto de partida y sirve como una suerte de guía que ayudará a comprender qué es la sustancia ${ }^{5}$.

Sin pretensión de agotar este concepto, nos limitaremos a indicar las dos notas características que tiene la definición leibniziana de sustancia como fuerza primitiva activa. En primer lugar, la sustancia se concibe como un sujeto o sustrato cuya esencia es la fuerza activa, es decir, la capacidad de pasar de un estado a otro cuando no hay impedimento para ello. En segundo lugar, Leibniz plantea que tal fuerza es la fuente de la que surgen las fuerzas derivativas, tales como las motrices, las cuales no serían sino modificaciones suyas. La fuerza primitiva es invariante, o sea, es una y la misma fuerza que se mantiene en las distintas tendencias particulares a acciones determinadas, las cuales no son sino sólo sus modificaciones. De este modo, la sustancia se piensa como una unidad invariante capaz de causar todos los distintos cambios de estado cuando no hay impedimento para ello o, en terminología leibniziana, fuerza activa primitiva.

Entre los distintos debates que emprende Leibniz en los años que siguen a sus primeras publicaciones sobre metafísica, la correspondencia con De Volder tiene cierto privilegio para abordar el problema de la noción leibniziana de sustancia. La principal ventaja que tiene esta discusión epistolar frente a las muchas otras que generaron las primeras publicaciones de Leibniz sobre

\footnotetext{
5 "A fin de ofrecer algún anticipo, diré por ahora que la noción de fuerza o potencia (que los alemanes llaman Kraft y los franceses force), a cuya explicación he dedicado una ciencia especial, la dinámica, aportará una grandísima luz para entender la verdadera noción de sustancia" (GP IV, p. 469).
} 


\section{Dossiê Leibniz, Dissertatio - Volume Suplementar 03 U UFPel [2016]}

filosofía primera radica en que el físico holandés, a diferencia de los otros objetores, pone en tela de juicio una de las tesis fundamentales de la metafísica leibniziana: la actividad primitiva u original de la sustancia corpórea. ${ }^{6}$ En particular, De Volder, insatisfecho con la explicación que Leibniz ofrece en sus textos, le pregunta por qué son necesarias fuerzas primitivas de actuar en la naturaleza. La cuestión que nos interesa es cómo procede Leibniz para defender la tesis según la cual sustancia es fuerza primitiva de actuar. Trataremos dos cuestiones. En primer lugar, evaluaremos si el argumento a priori de las fuerzas vivas que se desarrolla en la correspondencia constituye un argumento a favor de las fuerzas primitivas. En segundo lugar, estudiaremos la discusión en torno a la noción de sustancia en general en términos de fuerza primitiva activa y discutiremos qué tipo de argumentación Leibniz desarrolla.

\section{El debate sobre el argumento a priori de las fuerzas}

La correspondencia entre Leibniz y De Volder se inicia con algunas consideraciones críticas respecto de la prueba a posteriori, desarrollada en la Brevis demonstratio (1686), a favor de la distinción entre la cantidad de movimiento y la fuerza motriz y la correcta estimación de esta última. En el marco de este debate

${ }^{6}$ Para una presentación general de la figura de De Volder así como de la correspondencia con Leibniz, cf. Lodge 2005. Es importante advertir que el conocimiento que De Volder tiene de la metafísica leibniziana se reduce a los escritos publicados por Leibniz hasta 1698, esto es, De primae philosophiae emendatione (1694), el Système nouveau de la nature (1695), el Specimen dynamicum (1695) y De ipsa natura (1698). Ahora bien, además de estos textos, De Volder conoce fundamentalmente las tesis físicas de Leibniz; tanto la Brevis demonstratio (1686) como los debates con cartesianos como Papin y Catelan acerca de la medición de las fuerzas motrices. Es por ello que la discusión sobre metafísica va en paralelo al debate sobre física. Cf. GARBER 2009: 303-305. 
de filosofía natural, el físico holandés trae a colación un problema fundamental de la metafísica leibniziana:

\begin{abstract}
Si tuviéramos demostrado a priori que toda sustancia es activa, fácilmente me convencería de que de tan fecundísima fuente de verdades no sólo se seguiría el esclarecimiento de todos mis reparos, sino también de todas las dificultades que han agobiado a cuanto físico ha habido [...]. Porque, en efecto, una vez que conste que la naturaleza de la sustancia corpórea contiene la acción, parece lógico pensar que se mantendrá la misma mientras los cuerpos duren. ${ }^{7}$
\end{abstract}

El pedido de una demostración a priori de la actividad de la sustancia obliga a Leibniz a reconstruir el núcleo de su metafísica de los cuerpos. Respecto de este punto, en los últimos años intérpretes como Anne-Lise Rey (2009) sostienen que la respuesta leibniziana a ese interrogante puede comprenderse si tomamos como punto de partida la denominada prueba a priori de la conservación de la acción motriz ${ }^{8}$. A fin de evaluar esta tesis, expondremos en qué consiste el argumento a priori a favor de las fuerzas y cuál es su función en el debate con De Volder.

Consideremos primero algunos elementos mínimos del contexto en el que Leibniz presenta su prueba a priori. El reparo original de De Volder, que puede encontrarse ya en su correspondencia con Bernoulli (cf. GP II, p. 148-

\footnotetext{
${ }^{7}$ GP II, p. 151

${ }^{8}$ Cf. Rey (2009), p. 48. Sobre la base de los trabajos de Fichant (1997), que advierte la homonimia del término acción en el sistema leibniziano (el cual se utiliza tanto en física como en metafísica), Anne-Lise Rey plantea que, en verdad, se trata de un término ambivalente la medida que presenta información válida para la acción dinámica y asimismo para la acción metafísica. En particular, Rey cree que es en el concepto de acción formal, núcleo de la prueba a priori, donde se muestra la ambivalencia de la noción de acción y, por consiguiente, el punto de contacto entre la dinámica y la filosofía primera leibniziana. Es importante advertir que el propio Fichant ya había planteado que en la acción radicaba el puente entre ambas disciplinas (FICHANT 1998: 231).
} 


\section{Dossiê Leibniz, Dissertatio - Volume Suplementar 03 U UFPel [2016]}

152), está dirigido a uno de los pilares del argumento a posteriori ofrecido en la Breve demostración. Al igual que antes hicieran Catelan y Papin, el físico holandés critica el uso que Leibniz hace del axioma de Descartes y Pascal según el cual es necesaria la misma fuerza para elevar un cuerpo de cuatro libras a un pie que un cuerpo de una libra a cuatro pies. Del mismo modo que los otros cartesianos, De Volder sostiene que ese principio no vale universalmente, sino que depende de la velocidad de elevación y caída del cuerpo, esto es, que sólo es válido si la fuerza motriz de los cuerpos se estima en un mismo intervalo temporal. ${ }^{9}$ En el marco de este debate y, especialmente, de esta objeción recurrente de los cartesianos, Leibniz comienza a desarrollar un argumento a priori con el objeto de poner fin a la polémica de las fuerzas vivas. ${ }^{10}$ Ya en la primera carta y luego de responder a las críticas a la prueba a posteriori, el filósofo alemán añade:

\footnotetext{
[...] además, yo pruebo de manera sorprendente esta misma medida de las fuerzas con otras demostraciones a priori, a saber, por la naturaleza íntima de la acción y de la potencia, cosa que ahora omitiré aquí a fin de no extenderme demasiado y porque pienso que lo dicho posee suficiente entidad. ${ }^{11}$
}

El argumento propuesto por Leibniz se estructura y despliega de manera peculiar. La prueba a priori se desarrolla a partir de cinco conceptos fundamentales: además de la acción y la fuerza o potencia, reconocidos por Leibniz,

\footnotetext{
${ }^{9}$ Cf. Duchesneau (1994), p. 280.

${ }^{10}$ La primera mención de la prueba a priori se encuentra en el Dynamica de potentia (MS VI, 203). En los años que siguen, Leibniz debate con Bernoulli sobre la misma en 1696 (MS, p. 240-241, 312) y, posteriormente, se plantea a Papin -sobre la correspondencia con éste último, cf. Ranea (1989). El argumento a priori es expuesto a De Volder en la carta del 24 de marzo de 1699 (GP II, p. 168-175). 11 GP II, p. 158.
} 
requiere también los de espacio, tiempo y movimiento. Ahora bien, estas cinco nociones se suponen asimismo en la prueba a posteriori. La diferencia esencial entre estos dos argumentos se encuentra, empero, en los tipos de acción que entran en juego en cada una. En efecto, Leibniz distingue entre la acción violenta y la acción formal o libre. Por acción violenta entiende aquella cuyo ejercicio consume la fuerza del cuerpo a fin de vencer una resistencia exterior, tal como sucede, por ejemplo, en el ascenso y descenso de un cuerpo o en el choque. La acción formal, por el contrario, es aquella que se ejerce sin detrimento alguno de la potencia del cuerpo, tal como sucedería, por ejemplo, en el movimiento sin resistencia en un plano horizontal; se trata, en suma, de una acción libre de impedimentos externos. De este modo, la acción formal se diferencia de la violenta en la medida en que, por la ausencia de resistencia, el cuerpo conserva su fuerza a lo largo del movimiento.

La diferencia entre estos tipos de acciones importa a la hora de hacer a partir de ellas la medición de las fuerzas motrices. En el caso de la acción violenta, Leibniz sostiene que es irrelevante la consideración del tiempo, pues

[...] en una acción que consume su fuerza (o sea, que supera un impedimento al que ha de transferir su fuerza), esta fuerza debe medirse no por la duración, sino simplemente por el efecto, puesto que cualquiera que sea el tiempo que necesite, no podrá prestar un efecto mayor que la fuerza que tiene. ${ }^{12}$

12 GP I| 190. Leibniz resume su posición a Bernoulli del siguiente modo: "en las acciones violentas, o sea, en aquellas que consumen su potencia actuando frente a obstáculos, lo que ha de medirse es la cantidad, no la rapidez, sino simplemente el efecto real" (MS III 609) 


\section{Dossiê Leibniz, Dissertatio - Volume Suplementar 03 UFPel [2016]}

En otras palabras, la fuerza que hay detrás de la acción violenta ha de estimarse únicamente por la cantidad de efecto que produce hasta consumirse, sin importar el tiempo que demore en hacerlo. Sin embargo, si se quiere medir la potencia de un cuerpo a partir de su acción formal la situación es distinta. Por cierto, Leibniz advierte que, en ese caso, la medición de la fuerza se hace en función del efecto que se produce en un determinado tiempo. Tal como explica a Bernoulli, "en las acciones libres, o sea, en aquellas que meramente ejercitan su potencia, la acción y la fuerza de obrar deben medirse no sólo por el efecto sino también por su rapidez."13

Esta acción se piensa como "compuesta en razón de la potencia y del tiempo en que se ejerce". ${ }^{14}$ De este modo, Leibniz juzga que tiene más fuerza un cuerpo que realiza la misma acción formal en menor tiempo -tesis que constituye el núcleo en el debate de la prueba a priori ${ }^{15}$. Ahora bien, una vez trazada la diferencia que hay entre la estimación de la fuerza motriz a partir de la acción violenta y la acción libre, Leibniz advierte que en el segundo caso,

\footnotetext{
${ }^{13}$ MS III, p. 609. Leibniz explica la cuestión a De Volder en estos términos: "a esta acción, que aquí llamo libre, suelo a veces calificarla de formal, puesto que es natural al agente o fluye por sí misma de la naturaleza o estado de la cosa; por eso, ha de ser también continua, pues la interrupción provendría de otra parte; deberá, pues, entenderse como una acción puramente libre, no mezclada con lo violento, 0 sea, tal que no encuentre fuerza externa alguna o resistencia que tenga que superar; si no fuera así, es claro que no podría medirse la fuerza del agente por su sola duración, puesto que otro objeto resistente la moderaría y entonces ni la acción que ejerce violencia desde fuera, ni la libre, podría entenderse como el simple ejercicio de la potencia ni estaría en razón compuesta de la potencia y del tiempo en que la ejerce. Hay, pues, una gran diferencia entre el ejercicio que conserva la potencia y que fluye de ella espontáneamente y el uso que consiste en su consumo o destrucción por el conflicto exterior" (GP II, $p$. 190).

14 GP II, p. 90.

${ }^{15}$ Sobre este punto Leibniz distingue, apelando a vocabulario medieval, entre la extensión y la intensión de la acción (cf. GP II, p. 202-203). cf. Rey (2009), p. 160.
} 
"aunque no hay en la naturaleza ninguna acción sin obstáculos, separamos mediante una abstracción de la mente aquello que en la cosa es por sí mismo de aquello que se mezcla con las circunstancias externas". ${ }^{16}$ La medición de la fuerza que se hace sobre la acción formal o libre no sólo se caracteriza por operar sólo con conceptos, sino además por ser irreproducible en la experiencia. La razón de ello se encuentra en la tesis leibniziana según la cual la acción que se sigue de una fuerza derivativa surge por el choque y limitaciones de otros cuerpos. En un universo sin vacio toda acción implica siempre superación de obstáculos. Por ello, en la naturaleza toda acción es acción violenta; la libre o formal es algo abstracto o ideal. Esta característica, empero, no anula la posibilidad de que también pueda mostrarse a partir de ese tipo de acción particular cuál es la correcta estimación de la fuerza motriz y su distinción respecto de la cantidad de movimiento.

Expuestas estas consideraciones preliminares, se puede pasar ahora a la consideración de la prueba ofrecida por Leibniz en la carta 4 (24 de marzo de 1699). El sistema físico que se presupone, como hemos visto, es el de un cuerpo en movimiento rectilíneo uniforme en un plano horizontal sin resistencia alguna:

Resumiré, pues, el argumento. Para movimientos uniformes de un mismo cuerpo: [i] La acción que hace el doble en tiempo doble es doble que la acción que bace lo simple en tiempo simple. Por ejemplo, la acción de recorrer dos leguas en dos horas es el doble que la acción de recorrer una legua en una hora. Pues la acción primera contiene formalmente a ésta o la repite exactamente dos veces, al recorrer dos veces una legua en una hora. [ii] La acción que bace lo simple en tiempo simple es doble que la acción que hace lo simple en tiempo doble. Por

${ }^{16}$ GP II, p. 90. 


\section{Dossiê Leibniz, Dissertatio - Volume Suplementar 03 U UFPel [2016]}

ejemplo, la acción de recorrer una legua en una hora es doble que la acción de recorrer una legua en dos horas. Es decir, hace más aquel que produce más rápidamente el mismo efecto. $\mathrm{Y}$ asumo que las acciones que producen el mismo efecto están en razón directa de las velocidades o inversa de los tiempos y, por eso, la acción de recorrer la longitud a doble velocidad vale el doble que la de recorrer la misma a velocidad simple o, lo que es lo mismo, ésta última está contenida en valor dos veces en aquella. De aquí se sigue la conclusión, a saber, [iii] La acción que hace el doble en tiempo doble es cuádruple que la acción que hace lo simple en el mismo tiempo doble. Por ejemplo, la acción de recorrer dos leguas en dos horas es cuádruple que la acción de recorrer una legua en dos horas. Del mismo modo se demostrará que la acción que hace el triple es nueve veces más que la acción que en el mismo tiempo hace lo simple y generalizando, las acciones equitemporales son como los cuadrados de sus velocidades. Q. E. D. ${ }^{17}$

Sobre la validez de la prueba no nos pronunciaremos ${ }^{18}$. Nuestro interés radica únicamente en determinar si, concedido el argumento, puede constituirse como una prueba a priori de la actividad de la sustancia. Para ello es necesario trazar algunas precisiones respecto del objetivo y función de esta prueba.

En primer lugar, Leibniz no responde a la prueba de la actividad de la sustancia inmediatamente con el argumento a priori. De hecho, en su primera réplica (carta 2) sólo se pronuncia respecto de la prueba a posteriori. El argumento a priori se introduce en la carta 4, ante la insistencia de De Volder. En la carta 6 (23 de junio de 1699), luego de haber presentado su argumento a partir de la acción formal, declara que

Hasta que no hayamos concluido la medida de las fuerzas, no sé si es lo más conveniente sumergirnos en problemas más oscuros, como son los de la naturaleza de la substancia y de la extensión, en donde veo que nuestras

17 GP II, p. 173.

${ }^{18}$ Para una formulación básica del argumento, cf. Gueroult (1934), p. 125-127 y Duchesneau (1994), p. 290-300. 
respectivas nociones no son las mismas. Es mejor resolver unas pocas cosas que darle mil vueltas a muchas. ${ }^{19}$

Si bien es algo manifiesto, es importante no perder de vista que el objetivo de Leibniz con el argumento a priori consiste en establecer la correcta medición de la fuerza motriz $\left(m v^{2}\right)$ y su distinción respecto de la cantidad de movimiento cartesiana $(m|v|)$, es decir, tiene la misma función que la prueba a posteriori. En efecto, con ella busca mostrar que hay efectivamente fuerzas motrices en la naturaleza (esto es, fuerza derivativa activa). Ahora bien, ni Leibniz ni De Volder creen que el argumento a priori brinde información acerca de la actividad de la sustancia. Esto se evidencia por el hecho de que en la carta 8 , posterior al argumento a priori, el filósofo alemán advierte al holandés que no hay que precipitarse en los problemas metafísicos y que la medición de la fuerza motriz es sólo una guía que nos prepara para tratar las nociones tales como la de materia, el movimiento y la sustancia corpórea. ${ }^{20}$

En segundo lugar, el argumento a priori a favor de las fuerzas motrices no tiene privilegio alguno para comprender la noción de fuerza primitiva activa

\footnotetext{
19 GP II, p. 186.

${ }^{20}$ En la carta 8 Leibniz señala la necesidad de comenzar por la medición de la fuerza motriz para pasar a tratar los temas de metafísica: "He sabido por nuestro común amigo, el Sr. Bernoulli, que poner en claro la actividad de la substancia le parece a usted de mayor importancia que medir las fuerzas. Quizás sea cierto y apruebo su opinión. Sin embargo, a mí me ha parecido siempre que ésta es la puerta que nos permite pasar de las cosas a la verdadera metafísica, a fin de liberar poco a poco nuestro espíritu de las falsas nociones de la gente y de los cartesianos sobre la materia, el movimiento y la substancia corpórea, y comprender que de estas nociones no pueden derivarse las reglas de las fuerzas y de las acciones y que, o se acude a Dios como recurso, o habrá que entender en los cuerpos alguna cosa más profunda. Porque si una mente no preparada se adentra en aquel santuario donde puede contemplarse desde sus orígenes la inesperada naturaleza de la substancia y del cuerpo, es de temer que la oscuridad le ciegue por exceso de luz" (GP II, p. 195).
} 


\section{Dossiê Leibniz, Dissertatio - Volume Suplementar 03 I UFPel [2016]}

con la que Leibniz caracteriza a la sustancia. En este punto nos distanciamos de interpretaciones como las de Rey (2009) que encuentran en la noción de acción formal un elemento capaz de dar cuenta de la actividad particular que Leibniz exige a la sustancia. En efecto, Anne-Lise Rey sostiene que este tipo de acción tiene una ambivalencia en el pensamiento leibniziano, esto es, es válida tanto para la física como para la metafísica. A partir de ello, sostiene no sólo que la acción formal hace inteligible la actividad de la sustancia, sino incluso una tesis radical: que esta última encuentra en la primera su fundamento ${ }^{21}$. A fin de establecer esto, Rey afirma, primero, que la acción formal es la acción esencial de los cuerpos -y que es raíz de la acción violenta ${ }^{22}$ y, segundo, que la acción formal revela una capacidad productiva interna que permite aprehender la actividad sustancial23. Pero, ¿es lícita la conclusión general extraída por Rey? A fin de comprender por qué juzgamos que no, atendamos a los dos problemas que tienen las dos tesis sobre las que fundamenta su propuesta. Por una parte, es necesario advertir que la acción formal es idéntica a la acción violenta, pues la distinción entre ambas es sólo de razón. De hecho, la primera no es más que una abstracción que se hace a partir de la segunda; la diferencia es puramente extrínseca: la acción formal es la acción violenta liberada del yugo de los obstáculos externos. Por esta razón, la tesis de Anne-Lise Rey según la cual la acción formal es la acción esencial de

\footnotetext{
${ }^{21}$ En principio, las características de la acción libre podrían llevar a considerarla como un mejor candidato para trazar un puente hacia la actividad de la sustancia. La interpretación de Rey busca mostrar una doble conclusión: en primer lugar, que la acción formal hace inteligible la actividad de la sustancia; en segundo lugar, que la acción formal fundamenta la acción sustancial. Cf. Rey (2009), pp. 164, 166 y 168.

${ }^{22}$ Cf. Rey (2009), pp. 49 y 159.

${ }^{23}$ Cf. Rey (2009), pp. 159-160 y 171.
} 
los cuerpos y es la raíz de la acción violenta no tiene lugar en el pensamiento de Leibniz. Por el contrario, si quiere determinarse qué depende de qué, la relación sería la inversa, esto es, la acción violenta es aquello sobre lo cual se abstrae idealmente la acción formal. Por otra parte, la acción formal, al igual que la violenta, se piensa como el desarrollo de una potencia motriz, esto es, una fuerza derivativa. En efecto, en tal acción no se revela ninguna fuerza productiva interna que se asemeje a la actividad que Leibniz pide a las sustancias. Creemos que la confusión que conduce a la lectura de Anne-Lise Rey se debe a que en la acción formal, al no haber impedimento externo, la fuerza motriz no se consume, sino que se preserva en el tiempo. Esta conservación, no obstante, no es la que Leibniz exige para la sustancia, ni tampoco se trata de una actio in se ipsum. Por una parte, la acción formal, como también la violenta, consiste en el paso de un estado al siguiente, esto es, se entiende como causa próxima. La acción motriz es, por ello, algo momentáneo en el sentido de que sólo contiene en sí el cambio hacia el estado siguiente. La particularidad de la acción formal es que ese momento se conserva en el tiempo, lo que sólo puede suceder en un sistema sin resistencia, es decir, ideal ${ }^{24}$. La conservación que Leibniz exige para la sustancia es otra cosa: es un estado invariante que permanece frente a distintos cambios de estados. Por otra parte, la acción formal, al igual que la violenta, es

\footnotetext{
${ }^{24}$ Que la acción formal sea un estado que contenga el subsiguiente no la vuelve apropiada para comprender la acción sustancial. En efecto, también la acción violenta contiene el estado subsiguiente. El problema del pasaje es que toda acción física (formal o violenta) incluye sólo el estado subsiguiente (es momentáneo), mientras que la acción de la sustancia abarca todos los estados, tanto pasados como futuros.
} 


\section{Dossiê Leibniz, Dissertatio - Volume Suplementar 03 I UFPel [2016]}

externa. Nuevamente es la consideración aislada (y abstracta) de un cuerpo sin obstáculos lo que puede llevar a pensar que se trata de una acción interna. En suma, el concepto de acción formal es propio de la física y no tiene privilegios para comprender la acción sustancial -al menos, no más que la acción violenta.

En tercer lugar, las razones por las que se introduce el argumento $a$ priori no son teóricas, sino fundamentalmente coyunturales. Como se ha visto, este argumento es diseñado por Leibniz en el marco de su debate con los cartesianos. Con él se busca establecer la correcta estimación de la fuerza motriz y distinguirla de la cantidad de movimiento baciendo intervenir al tiempo, esto es, bajo la condición exigida por Catelan, Papin y el mismo De Volder, a saber, que se evalúe la fuerza motriz en movimientos con una misma duración. En esta línea y en relación con su prueba a priori, Leibniz advierte al físico holandés que:

\footnotetext{
Algunas de estas cosas ya se las dije entonces a Catelan, sugiréndole a la vez que está tan lejos (como él pensaba) de ser un obstáculo para mí la consideración del tiempo, que antes bien a partir de éste se demuestra mi teoría, pero por una razón muy distinta de la que él suponía, que es la que ahora le he confiado a usted; pues me parece usted persona capaz de comprender un análisis tan profundo y bello; a él, en cambio, y a la gente en general, se lo dije más bien velado. ${ }^{25}$
}

En efecto, es por la insistencia de De Volder que Leibniz decide exponer el argumento a priori, que arriba a la fuerza motriz a partir de movimientos equitemporales. El motivo contextual por el que se introduce esta prueba incluso ha hecho dudar a algunos intérpretes, tales como Gueroult (1967, 
pp. 118-119), acerca de la pertinencia del método a priori en el proyecto leibniziano de filosofía natural ${ }^{26}$.

Por estas razones puede observarse que con la introducción del argumento a priori Leibniz no busca fundamentar la actividad primitiva de la sustancia. En efecto, al igual que con el argumento a posteriori, su objetivo es establecer la distinción de la fuerza motriz respecto de la cantidad de movimiento. Sin embargo, este concepto propio de la física es propuesto por el filósofo alemán como guía para esclarecer la noción metafísica de sustancia, la cual, empero, tiene un fundamento independiente. Veamos entonces la defensa que Leibniz emprende de la definición de sustancia como fuerza primitiva activa.

4. La fuerza primitiva activa como hipótesis válida: la

\section{argumentación controversial}

Una vez aceptada, o al menos presentada ${ }^{27}$, la correcta medición de la fuerza motriz, Leibniz introduce su noción de sustancia. En relación con este tema, tal como plantea Lodge (1998), la correspondencia entre ambos pensadores constituye, sin duda, un fracaso. Ello se debe fundamentalmente a que la exigencia original de De Volder, a saber, el desarrollo de una prueba a

${ }^{26}$ Sobre la pertinencia del método a priori en la física leibniziana, cf. Gueroult (1967), pp. 118-119 y Duchesneau (1994), pp. 300-307. Es importante advertir, sin embargo, que Leibniz mismo valora positivamente el argumento: "Yo siempre he valorado más esta demostración, pues no utiliza la hipótesis del peso ni la de la elasticidad ni ninguna otra circunstancia, sino que fluye de forma transparente de las nociones primeras y abstractas" (GP II, p. 174).

${ }^{27}$ De Volder discute a lo largo de la correspondencia las pruebas leibnizianas a favor de la fuerza motriz, tanto la versión a posteriori como la a priori. En efecto, el debate sobre los temas de filosofía natural siguen en paralelo a la discusión sobre temas metafísicos. 


\section{Dossiê Leibniz, Dissertatio - Volume Suplementar 03 UFPel [2016]}

priori a favor de la actividad de la sustancia, no encuentra respuesta por parte de Leibniz, quien parece oscilar entre evadir la cuestión o directamente plantear que no se necesita prueba alguna ${ }^{28}$. A pesar de ello, el debate sobre la noción de sustancia tiene dos elementos que lo tornan capital para comprender la propuesta leibniziana: en primer lugar, profundiza la definición de sustancia como fuerza primitiva activa; en segundo lugar, enseña algunas características generales del modo de argumentación que Leibniz lleva adelante.

Como punto de partida de la discusión, Leibniz declara a De Volder que, a diferencia de Descartes, él ubica la esencia de la sustancia en general en una potencia o to dynamikon que, en la carta 4, es caracterizada del siguiente modo:

No hemos de buscar otra noción de potencia o fuerza más que la de ser un atributo del que se sigue la mutación, cuyo sujeto es la sustancia misma. No veo yo que esto se encuentre más allá de nuestra inteligencia [...]. Y no puede eliminarse este principio activo o fondo de actividades, puesto que las fuerzas activas son accidentales o mudables y los movimientos mismos son modificaciones de alguna cosa substancial; pero tales fuerzas y acciones no pueden ser modificaciones de una cosa meramente pasiva como es la materia. La conclusión es que debe darse un activo primero o substancial, que sea modificado por la presencia de un dispositivo pasivo o materia. Por lo tanto, las fuerzas secundarias o motrices y sus movimientos deben atribuirse a la materia segunda o cuerpo completo, que resulta de lo activo y lo pasivo. ${ }^{29}$

En esta definición, Leibniz retoma las dos ideas nucleares con las que caracteriza a la sustancia. En primer lugar, se trata de un sujeto o sustrato cuya esencia es la fuerza activa, es decir, la capacidad de pasar de un estado a otro 
cuando no hay impedimento para ello. En segundo lugar, Leibniz plantea que es la fuente de la que surgen las fuerzas secundarias, tales como las motrices, las cuales no serían sino modificaciones suyas. De este modo, la sustancia se piensa como una unidad invariante capaz de causar todos los distintos cambios de estado cuando no bay impedimento para ello o, en terminología leibniziana, fuerza activa primitiva. De hecho, Leibniz introduce la noción de fuerza primitiva activa en las cartas subsiguientes. Cabe señalar que De Volder comprende esta noción adecuadamente, esto es, como una fuerza que es causa de todas las fuerzas secundarias (motrices en el caso de los cuerpos) y que no aumenta ni disminuye con ellos, sino que se conserva como algo invariante. Asimismo, es consciente de que la conservación de la fuerza primitiva no es la de las fuerzas vivas (que plantea la conservación para un sistema de cuerpos y se refiere a fuerzas secundarias), sino que es algo más radical: es la conservación de un estado en la cosa misma que subyace a todos sus cambios.

Más complicado que precisar la definición de sustancia ofrecida es la de dirimir la cuestión acerca de si hay o no aquí un argumento. Leibniz parece esbozar una suerte de prueba o, al menos, así lo lee De Volder. La demostración leibniziana podría reducirse en tres pasos: (1) las fuerzas activas secundarias (como las fuerzas motrices) son modificaciones de una sustancia (sustrato común a ellas); (2) si el sustrato fuese pasivo, no se seguirían tales modificaciones; (3) entonces, el sustrato es activo. Este simple - y circular - 


\section{Dossiê Leibniz, Dissertatio - Volume Suplementar 03 I UFPel [2016]}

razonamiento es, como veremos, lo más cercano a una prueba a priori o de tipo deductiva a favor las fuerzas activas primitivas que hay en la correspondencia.

El trato amigable entre los corresponsales comienza a menguar desde la respuesta de De Volder, quien presenta las siguientes réplicas:

Me sorprende mucho esto que usted dice: no hemos de buscar otra noción de potencia o fuerza más que la de ser un atributo del que se sigue la mutación, cuyo sujeto es la sustancia misma. No veo yo - añade usted - en qué supere esto la capacidad de nuestra inteligencia. Yo, en cambio, no veo aquí nada que mi entendimiento capte. El sujeto de las mutaciones es una mera noción lógica que no explica nada [...] [Dice que] Las fuerzas activas mudables son modificaciones de alguna cosa sustancial. Sin duda. Pero no pueden serlo de una cosa meramente pasiva. Pero es que tal cosa pasiva no se da, si verdaderamente se muestra que toda sustancia es activa; pero justamente mi mayor dificultad radica en saber qué cosa sea esto. ${ }^{30}$

Sorprendido por la respuesta del filósofo alemán - lo que es comprensible en tanto el físico holandés lo conocía fundamentalmente por sus textos sobre física y matemática -, De Volder cuestiona dos puntos de la propuesta leibniziana. En primer lugar, rechaza la supuesta inteligibilidad de la tesis que afirma que la noción de potencia implica un sustrato "del que se sigue la mutación”, esto es, un sustrato activo. En efecto, el físico holandés es consciente de que si acepta eso, entonces ya concedió aquello que está buscando, a saber, que la sustancia es activa. De allí que califica a la definición leibniziana como una "noción lógica que no explica nada". Habiendo rechazado que se trate de algo manifiesto, exige en consecuencia su demostración. En segundo lugar, De 
Volder cuestiona el argumento presentado por Leibniz. Su crítica puede leerse como una acusación de petitio principii. En efecto, De Volder concede el paso 1, esto es, que hay sustratos de las modificaciones. Con ello se establece un doble punto de acuerdo: en primer lugar, que hay sustancias y, en segundo lugar, que hay fuerzas motrices (esta última concesión se hace en pos del debate sobre metafísica, pues la polémica sobre ellas y su necesidad en física es un tema sobre el que siguen polemizando en paralelo) ${ }^{31}$. El punto en discusión, entonces, radica en determinar si es necesario que el sustrato o sustancia de estos cambios sea algo activo. El problema que De Volder encuentra en el argumento leibniziano es que en el paso 2 se presupone ya lo que ha de probarse, a saber, la actividad de la sustancia (paso 3), pues un sustrato pasivo vuelve inexplicable la actividad de la sustancia únicamente si se presupone ya esta última. La circularidad del argumento es manifiesta - lo que podría hacer suponer que Leibniz no lo pensó como una prueba. Dados estos problemas, De Volder reitera hacia el final de su epístola nuevamente su pedido original, a saber, una prueba de la actividad de la sustancia.

\footnotetext{
${ }^{31}$ Tal como indica Garber (2009), p.307, De Volder - y los cartesianos en general - no sólo cuestionan la introducción de la noción metafisica de fuerza primitiva (paso radical de Leibniz) sino además el concepto mismo de fuerza motriz en física. En esta línea puede leerse, por ejemplo, esta crítica de De Volder: "Añade usted que, además de la extensión, se requiere una cierta fuerza [...] que, según usted, no cae bajo el ámbito de la imaginación sino sólo del entendimiento. Por mi parte, le diré que ni siquiera [esta noción] me entra en el entendimiento mientras no conozca la causa o fundamento de esas fuerzas. Cuando yo hablo de fuerzas pero no comprendo la causa de las fuerzas, no hablo más que del efecto, de manera que sólo percibiría las fuerzas mismas cuando conociera lo que son, de dónde fluyen y por qué razón producen necesariamente su efecto" (GP II, p.166). La concesión de las fuerzas motrices se hace en pos del debate metafísico sobre las fuerzas primitivas.
} 


\section{Dossiê Leibniz, Dissertatio - Volume Suplementar 03 U UFPel [2016]}

De aquí en más la discusión se torna áspera. En las cartas subsiguientes

Leibniz busca clarificar su concepto de fuerza primitiva. Con tal fin, en efecto, presenta una primera analogía entre estas fuerzas y las series numéricas:

Dice usted que el movimiento, esto es, lo que resulta de la masa y la velocidad, son fuerzas derivativas. Yo, en cambio, no considero al movimiento como fuerza derivativa, sino que pienso que el movimiento (es decir, la mutación) se sigue de ella. La fuerza derivativa es el estado mismo presente en tanto que tiende al siguiente o pre-envuelve al siguiente, en la medida en que todo lo presente está grávido de futuro. Pero lo persistente mismo, en cuanto que envuelve todos los casos, tiene fuerza primitiva, de manera que la fuerza primitiva es como la ley de una serie, y la fuerza derivativa es como la determinación que designa un término concreto en la serie. $^{32}$

En principio, Leibniz recuerda que la fuerza no es el cambio o mutación misma, sino que es el estado que tiende a ello. Con eso en mente, distingue entre las fuerzas primitivas y las derivativas. Mientras las segundas se caracterizan por su carácter momentáneo, esto es, por retener el estado inmediatamente anterior y tender sólo hacia el subsiguiente, las primeras se definen, en cambio, por envolver todos los estados - tanto pasados como futuros. De allí que Leibniz compare a la fuerza primitiva con la ley de una serie numérica en la medida en que es algo que contiene las infinitas determinaciones que se siguen de ella. ${ }^{33}$ Es importante notar que todas estas definiciones,

32 GP II, p. 262.

${ }^{33}$ Leibniz advierte en relación con este punto que es por esta fuerza invariante que puede explicarse que la misma sustancia sea aquella que persiste a los múltiples cambios y que de allí es de donde derivamos la idea de identidad de la sustancia. "La ley [de la serie] que es la que nos permite a nosotros formarnos una opinión de la identidad de un mismo sujeto modificado o mónada. Es la persistencia de una determinada ley, que implica los estados futuros de aquello que concebimos como idéntico, lo que constituye, en mi opinión, la identidad de la substancia" (GP II, p. 264). 
analogías y tesis vinculadas a la fuerza primitiva se encuentran ya en el período medio $^{34}$. La idea central en la correspondencia con De Volder es, como lo era entonces, que la esencia de la sustancia es tener una fuerza primitiva, esto es, una potencia invariante y común a la multiplicidad de fuerzas derivativas determinables en la naturaleza. En las cartas subsiguientes, Leibniz presenta una segunda analogía. Nuevamente con el interés de clarificar su concepto de fuerza primitiva, el filósofo alemán traza una comparación con la percepción y con el apetito que percibimos en nosotros mismos:

\begin{abstract}
Convendrá, pues, considerar ahora que en este principio de acción [la fuerza primitiva] hay mucho de inteligible, pues en él hay algo análogo a lo que reside en nosotros, a saber, la percepción y el apetito, ya que, al ser uniforme la naturaleza de las cosas, no puede ser la nuestra infinitamente distinta de todas las demás substancias simples de las que se compone todo el universo. Más aún, analizando esto con rigor, habrá que afirmar que nada hay en las cosas sino substancias simples, y en ellas la percepción y el apetito. ${ }^{35}$
\end{abstract}

Respecto de estas precisiones y analogías, De Volder es consciente de que con ellas no alcanza para responder a su inquietud original. En efecto, el físico holandés piensa que, incluso concediendo que hay fuerzas derivativas en la naturaleza, hay que dar razones para introducir algo tal como las fuerzas primitivas. Es en función de ello que De Volder dirige al planteo leibniziano dos clases de objeciones. En primer lugar, indica que la actividad primitiva de las sustancias no es algo que se necesite en filosofía natural. Por el contrario, en

\footnotetext{
${ }^{34}$ La analogía matemática no es nueva en la filosofía de Leibniz; se encuentra ya en el período parisino, cf. AA VI 3, p. 326. Tampoco lo es ni la definición de fuerza primitiva y derivativa, ni el vínculo con el problema de la identidad del yo, tema que se discute, por ejemplo, con Arnauld. 35 GP II, p. 270.
} 


\section{Dossiê Leibniz, Dissertatio - Volume Suplementar 03 I UFPel [2016]}

física alcanza - o incluso sobra a juicio de De Volder - con la conservación de

las fuerzas derivativas para dar cuenta por completo de las leyes del movimiento y todo cambio en la naturaleza. La introducción de una fuerza primitiva en cada cuerpo, invariante e indivisible, es en filosofía natural una hipótesis gratuita que no explica nada. ${ }^{36}$ Es importante notar que Leibniz comparte esta tesis: en física basta con la conservación total de las fuerzas secundarias y no se requiere de fuerzas primitivas. En segundo lugar, De Volder plantea su crítica más fuerte, a saber, que tampoco estas últimas fuerzas son necesarias en metafísica. Sobre este punto no se afirma que se trate de algo imposible, sino sólo que no es la única explicación posible. En este punto De Volder se alinea con el principal enemigo de la metafísica leibniziana en estos años: el ocasionalismo. En efecto, otra opción válida es pensar que Dios, en lugar de crear fuerzas primitivas como quiere Leibniz, directamente introduzca las fuerzas derivativas en la naturaleza. Y como la experiencia sólo enseña los cambios o mutaciones, pero no sus causas, se requiere de una argumentación adicional para determinar si efectivamente la explicación correcta es la de Leibniz o la de sus contrincantes. ${ }^{37}$

\footnotetext{
${ }^{36}$ De Volder presenta el reparo en estos términos: "Responderá usted quizás que, aunque se modifiquen estas que usted gusta llamar fuerzas derivadas, la fuerza primitiva permanece la misma. Lo cual yo lo entendería diciendo que permanece la misma fuerza total que se encuentra en todos los cuerpos tomados en conjunto, y que se distribuye de forma variada. Pero lo que no acabo de ver claro es cómo permanece la misma fuerza en este cuerpo concreto, cuando se activan los órganos por los que se ejercen sus fuerzas. Si suponemos que en cada cuerpo no residen más que las fuerzas que ya tienen y que usted llama derivadas, ¿no será esto suficiente para que de aquí se siga todo lo que haya de ocurrir en toda la naturaleza de las cosas? ¿Qué necesidad tenemos de establecer, además, unas fuerzas primitivas, y que además sean indivisibles?" (GP II, p. 244).

${ }^{37}$ La posible salida ocasionalista es expuesta por De Volder del siguiente modo: "Así pues, si suponemos que Dios al principio de las cosas introdujo en la materia tantas fuerzas derivativas cuantas ésta tiene ahora, ¿qué necesidad hay de acudir a esa tendencia intrínseca de las cosas a la mutación? En mi hipótesis, o los cuerpos particulares no serían substancias, si para que haya substancia usted desea esa
} 
De este modo, De Volder pregunta cuál es la razón por la que ha de preferirse su sistema al de Malebranche. ${ }^{38}$ En conclusión, dado que la fuerza primitiva no se necesita en física y en metafísica no es la única opción viable, el físico holandés juzga necesario la presentación de alguna demostración a favor de la tesis leibniziana sobre la actividad primitiva como algo ínsito en las sustancias.

El pedido de De Volder por una prueba de la actividad de la sustancia está adecuadamente fundado. Sin embargo, la respuesta por parte de Leibniz nunca llega o, mejor dicho, no lo hace al menos en los términos que exige el holandés, a saber, como una prueba a priori. La disconformidad con la respuesta leibniziana se percibe incluso en su última intervención, donde declara que: "hay algo en mí que hasta este momento me hace dudar sobre las mismas dificultades

unidad o fuerza primitiva, o, si las llamamos substancias, serían substancias de la misma naturaleza, donde ningún inconveniente habría en que unas actuaran sobre otras. Yo sé que para suponer estas fuerzas derivadas hay que acudir a Dios; pero también usted lo hace para las primitivas. Y no por ello dejaríamos de decir que Dios ha producido en las cosas aquellos principios de mutaciones que permiten inferir los hechos posteriores de los anteriores: siempre habría producido Dios fuerzas derivadas, de las que se seguiría toda mutación. La experiencia enseña, sin duda, que se producen mutaciones; pero lo que entre usted y yo nos preguntábamos no era qué es lo que la experiencia enseña, sino qué es lo que se seguiría a priori de la sola naturaleza de las cosas; y la verdad es que ninguna experiencia, que yo sepa, enseña que emanen desde dentro. En cuanto a los procesos de la mente, son todavía más oscuros como para que de ellos podamos extraer argumento. La conciencia enseña que se produce mutación en la mente, pero ocurre muchas veces de manera que el agente no tiene consciencia de la causa exterior de sus procesos. De manera que suponer que aquella mutación depende de la naturaleza intrínseca de la mente me parece a mí una suposición exagerada" (GP II, p. 260-261)

${ }^{38}$ De Volder no se muestra partidario de Malebranche. Incluso reconoce la mayor simplicidad de la propuesta de Leibniz. El problema es que no ve razones en pos de uno u otro, de allí que afirme: "Sin duda, yo prefiero su hipótesis a la de Malebranche, aunque no sea más que por el hecho de que él necesita de Dios para cada acto, y usted en cambio, sólo lo necesita en el primer acto físico de la producción. Pero hay algo que en ambos casos a mí me parece común, y es que la razón, ya sea de la primera producción de entelequias ya sea de la acción divina en cada una de ellas, permanece muy oscura y, además, ninguna de las dos hipótesis puede ser refutada por las leyes mecánicas, puesto que ambas las suponen. Hay, además, en su causa, algo que a mí me sienta mal, y es que supone de forma gratuita que toda substancia es activa, puesto que en la definición misma del término substancia usted incluye ya el principio de la acción" (GP II, pp. 254). En conexión con este problema es interesante notar que Leibniz parece leer a De Volder no tanto como un ocasionalista, sino como una spinozista. Sobre el posible cripto-spinozismo en la posición de De Volder, cf. Lodge (2005) y Garber (2009), pp. 307- 310. 


\section{Dossiê Leibniz, Dissertatio - Volume Suplementar 03 I UFPel [2016]}

que ya le expuse a usted, pues me parece que sigo sin entender esa fuerza primitiva". ${ }^{39}$ Este pasaje resume el fracaso de la correspondencia en lo que hace al pedido de una demostración a priori de la actividad de la sustancia.

Pero, ¿por qué Leibniz no presenta a De Volder un argumento a prior? La respuesta es simple: porque no lo tiene. En este punto es conveniente tener en cuenta que, si bien el pedido general del físico holandés está bien fundado, pues, independientemente de la verosimilitud o inverosimilitud de las fuerzas primitivas, al no tratarse de algo evidente, su verdad necesita de prueba, de allí no se sigue que tal argumento haya de ser a priori. Leibniz es consciente de su falencia en este punto. De hecho, en su primera carta a De Volder y como antesala a la discusión sobre la sustancia ya advierte:

Ojalá pudiera yo exponerle a usted o tuviera en este momento elaborada mis meditaciones sobre la naturaleza de la sustancia y todo lo que de ella se deriva con la misma claridad con que tengo ya la parte matemática de mi dinámica. ${ }^{40}$

Si bien Leibniz reconoce que sus argumentos a favor de las fuerzas primitivas no son comparables a los ofrecidos en dinámica, plantea que eso, empero, no anula la validez de su propuesta: "Finalmente, aun concediendo que no podemos tener una demostración a priori de todo, tal como usted me pide, ¿es que por ello mi bipótesis concuerda menos con las cosas?". ${ }^{41}$ En la misma

\footnotetext{
${ }^{39} \mathrm{GP}$ II, p. 279.

40 GP II, p. 162.

41 GP II, p. 241.
} 
línea Leibniz explica su posición a Bernoulli mostrando, a su vez, su disconformidad respecto del debate con De Volder:

\begin{abstract}
Al polemizar con el Sr. De Volder necesito tu ayuda tanto más cuanto más difícil es demostrar cosas novedosas [...] Yo no me niego a responder a sus objeciones, pero dar demostraciones de todo no sería propio ni de este lugar ni de esta ocasión, aunque las tuviera a mano [...]. Yo creí que me bastaría con ofrecer mi pensamiento como una hipótesis ante los que creo jueces ecuánimes. ${ }^{42}$
\end{abstract}

A partir de estos pasajes puede observarse que Leibniz reconoce que, ante la falta de prueba a priori, la fuerza primitiva ha de pensarse como una hipótesis $^{43}$. Sin embargo, no cree que ello impida el debate sobre su validez. En efecto, el filósofo alemán juzga que, a pesar de su carácter hipotético, su tesis tiene ventajas sobre las otras hipótesis posibles en tanto es capaz de explicar de un modo más simple los principales problemas que aquejan a la metafísica, tales como la unión alma-cuerpo y su interacción, la identidad del yo, la libertad de las criaturas y la responsabilidad frente sus acciones. En efecto, es frecuente en sus presentaciones sobre temas de metafísica durante estos años que Leibniz afirme que, si bien su propuesta es, en definitiva, una hipótesis sobre la que no tiene demostración apodíctica, es, empero, la mejor hipótesis ofrecida hasta el momento en tanto explica los mismos problemas con menores supuestos. Por ejemplo, en una carta a Des Billettes de 1696 ya puede observarse el

42 MS III, p. 592.

${ }^{43}$ En referencia a sus tesis metafísicas, Leibniz recuerda a Bernoulli: "hasta ahora he planteado a modo de hipótesis algunas cosas cuya demostración exigiría un tratamiento mayor; de manera que, por el momento, las explicaciones y definiciones pueden servir de demostraciones, con tal de que se ajusten a los fenómenos" (MS II, p. 560). 


\section{Dossiê Leibniz, Dissertatio - Volume Suplementar 03 I UFPel [2016]}

reconocimiento por parte de Leibniz de los límites argumentativos de su propuesta: "Mi sistema, sobre el cual usted expresó curiosidad, no es un cuerpo completo de filosofía. Y no pretendo haber dado razón de todo lo que otros han buscado explicar. Debemos proceder por etapas y comenzar con principios, y espero ser capaz de satisfacer la mayoría de las dudas". ${ }^{44}$ Leibniz juzga en efecto que su hipótesis soporta mejor las críticas que las propuestas de Malebranche o Spinoza. En este sentido afirma a De Volder lo siguiente:

Yo siempre estaré dispuesto a resolver honradamente todas las dudas que se opongan a lo que afirmo, tal como constantemente lo vengo haciendo hasta ahora, cosa que seguramente esperaría usted en vano conseguirlo de la hipótesis contraria. 45

Es importante notar que, aun cuando podría pensarse una posible defensa fundada en los métodos de controversias - pensados originalmente para sus debates teológicos-, Leibniz nunca desarrolla un argumento en estos términos. ${ }^{46}$

De este modo, creemos que es necesario repensar el modelo argumentativo que desarrolla Leibniz en este caso, pues, tal como se evidencia a partir de las fuentes, la validez de la hipótesis de la fuerza primitiva se funda en la superioridad que guarda respecto de las opciones que han ofrecido sus contrincantes, lo que no implica, sin embargo, la posibilidad de cambiar de

${ }^{44}$ GP VII, p. 451.

45 GP II, p. 241.

${ }^{46}$ Sobre el método de controversias en Leibniz, cf. Dascal (2006) XXXVII-L. 
posición si se le enseñara alguna mejor. Los límites que reconoce en su argumentación metafísica son confesados explícitamente a De Volder:

Mi deseo habría sido explicarle todo con más precisión y probarlo con más solidez; pero en esta infancia en la que todavía vive nuestra filosofía ya es algo decir cosas que no parece puedan refutarse y deducir todo lo demás partiendo de unas pocas hipótesis no desdeñables. Quizás algún día podamos ir más lejos, si puedo contar sobre todo con la luz de su talento.47

Por ello, a pesar haber jugado quizás algún movimiento más que Descartes, Leibniz también termina aquí abandonando la partida, cuya continuación habrá de quedar a las generaciones futuras.

\section{Ediciones de los escritos de Leibniz}

LEIBNIZ, G. W. (1875-1890). Die philosophischen Schriften. Gerhardt, C. I (ed), 7 vols. Berlín.

(1848-1863). Mathematische Schriften, Gerhardt, C. I. (ed.), 7

vols. Berlin.

(2011). Obras filosóficas y cientificas. XVI: Correspondencia III.

Orio de Miguel, B. (ed.). Granada: Comares Editorial.

(1923-). Sämtliche Schriften und Briefe. Berlin-

Brandenburgischen Akademie der Wissenschaften und der Wissenschaften in Göttingen (ed.). Darmstadt-Leipzig- Berlin: Akademie Verlag.

47 GP II, p. 185. 


\section{REFERENCIAS BIBLIOGRÁFICAS}

DASCAL, M. (2006). G. W. Leibniz: The Art of Controversies. Dordrecht: Springer. DUCHESNAU, F. (1994). La dynamique de Leibniz. Paris : Vrin.

FICHANT, M. (1997). “Actiones sunt suppositorum: l'ontologie leibnizienne de l'action". Philosophie $\mathrm{N}^{\circ}$ 53: 135-148.

(1998). "De la puissance à la action: la singularité stylistique de

la dynamique", en Fichant, M. (ed.). Science et métaphysique dans Descartes et Leibniz.

Paris: PUF: 205-244.

GARBER, D. (2009). Leibniz: Body, Substance, Monad. Oxford: Oxford University Press.

GUEROULT, M. (1934). Dynamique et métaphysique leibniziennes. Paris : Belles Lettres.

LODGE, P. (2005). "Burchard de Volder: Crypto-Spinozist or Disenchanted Cartesian?”. SCHMALTZ, T. (ed). Receptions of Descartes: Cartesianism and AntiCartesianism in Early Modern Europe. Routledge: 128-146. (2004). "Leibniz's Close Encounter with Cartesianism in the

Correspondence with De Volder". LODGE, P. (ed.). Leibniz and His Correspondents, Cambridge: Cambridge University Press: 162-192.

(1998). “The Failure of Leibniz's Correspondence with De

Volder". Leibniz Society Review 8: 47-67.

MOLL, K. (1978-1996). Derjunge Leibniz. 3 tomos. Stuttgart: Bad Cannstatt. 
RANEA, G. (1989). "The a priori Method and the actio Concept Revised. Dynamics and Metaphysics in an Unpublished Controversy between Leibniz and Denis Papin”. Studia Leibnitiana XXI: 42-68.

REY, A.-L. (2009). "L'ambivalence de la notion d'action dans la Dynamique de Leibniz. La correspondence entre Leibniz et De Volder". En Studia Leibnitiana 41:1, pp. 47-66 y 41:2, pp.157-182. (2010). "The Controversy between Leibniz and Papin: from the Public Debate to the Correspondence". DASCAL, M. (ed.). The Practice of Reason. Leibniz and his Controversies. Amsterdam/Philadelphia: John Benjamins Publishing Company: 75-100.

SHIMONY, I. (2010). "Leibniz and the vis viva Controversy". DASCAL, M. (ed.). The Practice of Reason. Leibniz and his Controversies, Amsterdam/Philadelphia: John Benjamins Publishing Company: 51-74.

E-mail: rodolfofazio@gmail.com 